\title{
GROWTH AND OSCILLATION PROPERTIES OF SOLUTIONS OF A FOURTH ORDER LINEAR DIFFERENCE EQUATION
}

\author{
JOHN W. HOOKER AND WILLIAM T. PATULA ${ }^{1}$
}

(Received 18 January 1983; revised 2 December 1983)

\begin{abstract}
For the fourth-order linear difference equation $\Delta^{4} u_{n-2}=b_{n} u_{n}$, with $b_{n}>0$ for all $n$, generalized zeros are defined, following Hartman [5], and two theorems are proved concerning separation of zeros of linearly independent solutions. Some preliminary results deal with non-oscillation and asymptotic behavior of solutions of this equation for various types of initial conditions. Finally, recessive solutions are defined, and results are obtained analogous to known results for recessive solutions of second-order difference equations.
\end{abstract}

\section{Introduction}

In a recent paper [5] Hartman introduced the concept of a generalized zero of a solution of an $n$th order linear difference equation and discussed disconjugacy and non-oscillation of solutions of such equations. In this paper we consider generalized zeros and oscillation and asymptotic properties of solutions of fourth-order linear difference equations. In particular, we discuss discrete analogues for certain results of Leighton and Nehari [8] on oscillation and separation of zeros of solutions of a fourth-order differential equation.

Specifically, we consider here the fourth-order linear difference equation

$$
\Delta^{4} u_{n-2}=b_{n} u_{n}, \quad n=2,3,4, \ldots,
$$

where $b=\left\{b_{n}\right\}, n=2,3,4, \ldots$, is a given infinite sequence of positive numbers, $\Delta$ is the forward difference operator defined as $\Delta u_{n}=u_{n+1}-u_{n}$, and

$$
\begin{aligned}
& \Delta^{2} u_{n}=\Delta\left(\Delta u_{n}\right)=u_{n+2}-2 u_{n+1}+u_{n}, \\
& \Delta^{3} u_{n}=\Delta\left(\Delta^{2} u_{n}\right)=u_{n+3}-3 u_{n+2}+3 u_{n+1}-u_{n}, \\
& \Delta^{4} u_{n}=\Delta\left(\Delta^{3} u_{n}\right)=u_{n+4}-4 u_{n+3}+6 u_{n+2}-4 u_{n+1}+u_{n} .
\end{aligned}
$$

\footnotetext{
${ }^{1}$ Department of Mathematics, Southern Illinois University, Carbondale, Illinois 62901, U.S.A.

(C) Copyright Australian Mathematical Society 1985, Serial-fee code 0334-2700/85
} 
By a solution of (1) we mean a real sequence $u=\left\{u_{n}\right\}, n=0,1,2, \ldots$, which satisfies (1.1).

One possible application of our results would be in the numerical approximation of solutions of the fourth-order differential equation $y^{\prime \prime \prime}(t)-b(t) y(t)=0$, $b(t)>0$. This follows from the fact that the operator given by (1.1) is stable in the sense defined by Dahlquist [1, page 19], where the $h$ in [1, page 6] can be thought of as 1 . Another possible application lies with the numerical approximation of recessive type solutions discussed in Section 5. In the second-order case, the concept of recessive solutions has been important in the numerical approximation of certain types of special functions ([9]).

Difference equations also arise directly in mechanics (see Fort [2] and Greenspan [4]), in biology ([3]), and in the theory of codes ([12]), although these are more typically first and second order difference equations.

Before proceeding, we note that the letters $i, j, m, n, M, N, P, Q, R$ will be used below only as nonnegative integer variables. We occasionally write WLOG for "without loss of generality". By the graph of a solution $u$ of (1.1) we will mean the graph consisting of the line segments connecting successive points $\left(n, u_{n}\right)$ and $\left(n+1, u_{n+1}\right), n=0,1,2, \ldots$

In Section 2 we define generalized zeros and discuss uniqueness of solutions of (1.1) satisfying certain initial value properties. In section 4, several theorems culminating in two separation of zeros theorems are presented. Theorems 4.3 and 4.4 are discrete analogues of Theorems 2.6 and 3.1 of Leighton and Nehari [8], and the sequence of Lemmas in Section 3 is somewhat analogous to the corresponding chain of results of Leighton and Nehari.

In Section 5 we discuss existence of positive decreasing solutions (recessive solutions) of (1.1).

\section{Definitions and preliminary results}

It is readily verified from (1.2) that the fourth-order forward difference operator may be written in the form

$$
\Delta^{4} u_{n-2}=u_{n+2}-u_{n+1}-\Delta u_{n}-\Delta^{2} u_{n-1}-\Delta^{3} u_{n-2},
$$

and so (1.1) may be written as

$$
u_{n+2}=\Delta^{3} u_{n-2}+\Delta^{2} u_{n-1}+\Delta u_{n}+u_{n+1}+b_{n} u_{n} .
$$

Also, from (1.2) we may write (1.1) in the alternate form

$$
u_{n+4}=4 u_{n+3}-\left(6-b_{n+2}\right) u_{n+2}+4 u_{n+1}-u_{n}, \quad n \geqslant 0 \text {. }
$$


From (2.3) it follows inductively that all solutions of (1.1) are defined for all $n \geqslant 0$, and that a unique solution of (1.1) is determined if any four successive values $u_{N}, u_{N+1}, u_{N+2}, u_{N+3}$ are given. In particular, the following uniqueness property is clear.

LEMMA 2.1. If $u$ is a solution of (1.1) with $u_{N}=u_{N+1}=u_{N+2}=u_{N+3}=0$ for some $N \geqslant 0$, then $u_{n}=0$ for all $n \geqslant 0$.

We now define a "generalized zero" for a solution of (1.1) as follows:

Definition. Let $u=\left\{u_{n}\right\}, n=0,1,2, \ldots$, be a solution of (1.1). Then $n \geqslant 0$ is a generalized zero if one of the following holds:

$$
\begin{aligned}
& u_{n}=0 \text {; } \\
& n>0 \text { and } u_{n-1} u_{n}<0 \text {; } \\
& n>1 \text {, }
\end{aligned}
$$

and there exists an integer $k, 1<k \leqslant n$, such that

$$
(-1)^{k} u_{n-k} u_{n}>0 \text {, and } u_{n-1}=u_{n-2}=\cdots=u_{n-k+1}=0 \text {. }
$$

This is essentially the definition given by Hartman [5]. To this we add the following terminology:

A generalized zero of a solution $u$ of (1.1) is said to be of order 0,1 , or $k>1$, according to whether condition (2.4), (2.5), or (2.6), respectively, holds. In particular, a generalized zero of order 0 will simply be called a "zero", and a generalized zero of order one will be called a "node", as in Fort [2] and Hartman [5].

We note also that if $u$ has a generalized zero of order $k$ at $n$, then $u$ necessarily has $k$ consecutive generalized zeros at $n-k+1, \ldots, n$. Figure 1 illustrates generalized zeros of order $0,1,2$ and 3 respectively, at $n$. It will be shown below in Theorem 4.1 that a solution of (1.1) cannot have a generalized zero of order $k>3$. We know already from Lemma 2.1 that a nontrivial solution of (1.1) can have zeros at no more than three consecutive values of $n$. However, a solution of (1.1) can have arbitrarily many consecutive nodes, as exhibited by the sequence $u_{n}=(-1)^{n}$, which is a solution of $\Delta^{4} u_{n-2}=16 u_{n}$.
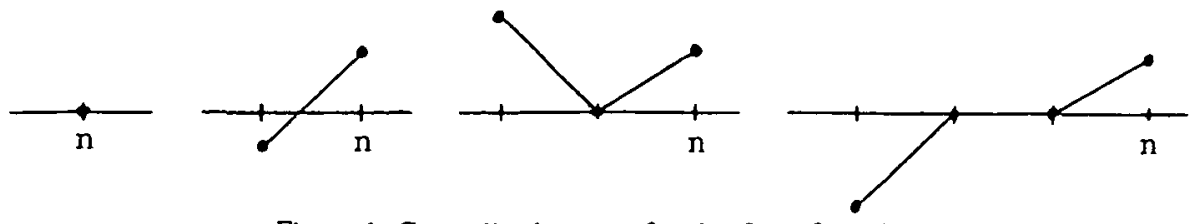

Figure 1. Generalized zeros of order $0,1,2$, and 3. 


\section{Monotonicity properties}

We begin with a preliminary lemma on the behavior of a sequence with a nonnegative second difference.

LEMMA 3.1. If $u$ is an infinite sequence satisfying $\Delta^{2} u_{n} \geqslant 0$ for all $n \geqslant K$ for some $K$, then

$$
u_{n} \geqslant u_{K}+\Delta u_{K}(n-K), \text { for all } n \geqslant K
$$

PRoof. Since $\Delta^{2} u_{n} \geqslant 0$ for $n \geqslant K, \Delta u_{n}$ is nondecreasing so

$$
u_{n}-u_{K}=\sum_{i=K}^{n-1} \Delta u_{i} \geqslant(n-K) \Delta u_{K}
$$

Our second lemma is a difference equation analogue of Lemma 2.1 of Leighton and Nehari [8]. Note that it is assumed here and throughout this paper that $b_{n}>0$ for all $n$.

LEMMA 3.2. If $u$ is a nontrivial solution of (1.1) and if

$$
\begin{aligned}
& \text { (a) } u_{n} \geqslant 0, \\
& \text { (b) } \Delta u_{n} \geqslant 0, \\
& \text { (c) } \Delta^{2} u_{n-1} \geqslant 0, \\
& \text { (d) } \Delta^{3} u_{n-2} \geqslant 0,
\end{aligned}
$$

for some $n=N, N \geqslant 2$, then (3.2) holds for all $n \geqslant N$, with strict inequality in (3.2a) for all $n \geqslant N+2$, strict inequality in (3.2b) for all $n \geqslant N+1$, and strict inequality in (3.2c) and (3.2d) for all $n \geqslant N+3$. Furthermore

$$
\Delta^{4} u_{n-2} \geqslant 0, \text { for all } n \geqslant N,
$$

with strict inequality for all $n \geqslant N+2$, and $u_{n}, \Delta u_{n}$, and $\Delta^{2} u_{n}$ all tend to $+\infty$ as $n \rightarrow \infty$.

Proof. Given $u_{N} \geqslant 0, \Delta u_{N} \geqslant 0, \Delta^{2} u_{N-1} \geqslant 0$, and $\Delta^{3} u_{N-2} \geqslant 0$, we immediately have $u_{N+1}-u_{N}=\Delta u_{N} \geqslant 0$, hence

$$
u_{N+1} \geqslant u_{N} \geqslant 0 \text {. }
$$

From (2.2) we obtain

$$
\Delta u_{N+1}=u_{N+2}-u_{N+1}=\Delta^{3} u_{N-2}+\Delta^{2} u_{N-1}+\Delta u_{N}+b_{N} u_{N} .
$$


Then (3.3) gives us

$$
\Delta^{2} u_{N}=\Delta u_{N+1}-\Delta u_{N}=\Delta^{3} u_{N-2}+\Delta^{2} u_{N-1}+b_{N} u_{N},
$$

and this in turn leads to

$$
\Delta^{3} u_{N-1}=\Delta^{2} u_{N}-\Delta^{2} u_{N-1}=\Delta^{3} u_{N-2}+b_{N} u_{N} .
$$

All the terms on the right-hand sides of (3.3), (3.4), and (3.5) are nonnegative, so $\Delta u_{N+1}, \Delta^{2} u_{N}$, and $\Delta^{3} u_{N-1}$ are nonnegative. Thus, assuming that conditions (3.2a-d) hold for $N$ implies that they also hold for $N+1$. Proceeding in this way, we see by mathematical induction that conditions (3.2a-d) hold for all $n \geqslant N$. Condition (3.2e) follows immediately from (1.1), since $b_{n}>0$ and $u_{n} \geqslant 0$ for all $n \geqslant N$.

Since $b_{N}>0$, at least one term on the right in (3.3) must be positive, since otherwise $u_{N+1}=u_{N}=u_{N-1}=u_{N-2}=0$ by (1.2), hence $u$ would be the trivial solution of (1.1) by Lemma 2.1. Thus $\Delta u_{N+1}>0$ and it follows that strict inequality holds in (3.2b) for all $n \geqslant N+1$. This implies that $u_{n}$ is strictly increasing for $n \geqslant N+1$, and since $u_{N+1} \geqslant 0$, we then have $u_{n}>0$ for all $n \geqslant N+2$, as claimed. This in turn implies, by use of (1.1), that strict inequality holds for (3.2e) for $n \geqslant N+2$. Also, using (3.4) and (3.5), we may conclude that $\Delta^{2} u_{n}>0$ and $\Delta^{3} u_{n-1}>0$ for all $n \geqslant N+2$. Therefore strict inequality holds in (3.2c) and (3.2d) for all $n \geqslant N+3$.

To prove the last statement in the conclusion, we observe that the sequences $\left\{u_{n}\right\},\left\{v_{n}\right\}=\left\{\Delta u_{n}\right\}$, and $\left\{w_{n}\right\}=\left\{\Delta^{2} u_{n}\right\}$ all satisfy the hypotheses of Lemma 3.1 for any $K \geqslant N$. Applying Lemma 3.1 to these three sequences yields, respectively,

$$
\begin{aligned}
u_{n} & \geqslant u_{K}+\Delta u_{K}(n-K), \\
\Delta u_{n} & \geqslant \Delta u_{K}+\Delta^{2} u_{K}(n-K), \\
\Delta^{2} u_{n} & \geqslant \Delta^{2} u_{K}+\Delta^{3} u_{K}(n-K),
\end{aligned}
$$

for all $n \geqslant K$. In particular, these inequalities are true for $K=N+2$, and since $\Delta u_{K}, \Delta^{2} u_{K}$, and $\Delta^{3} u_{K}$ are all positive for $K=N+2$, it follows that $u_{n}, \Delta u_{n}$, and $\Delta^{2} u_{n}$ tend to $+\infty$ as $n \rightarrow \infty$, which completes the proof.

We note that the above theorem fails if the hypothesis $u_{N} \geqslant 0$ is replaced by $u_{N+1} \geqslant 0$. This is shown by the example $u_{n}=(-1)^{n+1}$, which is a solution of $\Delta^{4} u_{n-2}=16 u_{n}$. This sequence satisfies $u_{3}=1>0, \Delta u_{2}=2>0, \Delta^{2} u_{1}=4>0$, $\Delta^{3} u_{0}=8>0$, but fails to have any of the monotonicity properties of the conclusion of the lemma. It is interesting to note also that the initial conditions given in the hypotheses of the lemma are not necessarily of "convex" type, i.e., the graph of a solution satisfying these conditions is not necessarily convex 
upward on the interval from $N-2$ to $N+1$. For example, a sequence $u$ satisfying $u_{0}=0, u_{1}=1, u_{2}=0, u_{3}=1$ satisfies the hypotheses of the lemma, since $u_{2}=0, \Delta u_{2}=1>0, \Delta^{2} u_{1}=2>0$, and $\Delta^{3} u_{0}=4>0$. However, the "convex" conditions $u_{0}=2, u_{1}=0, u_{2}=0, u_{3}=1$, do not, since in this case $u_{2}=0$, $\Delta u_{2}=1, \Delta^{2} u_{1}=1$, but $\Delta^{3} u_{0}=-1$.

The following lemma is a corollary of Lemma 3.2. It is essentially a special case of Lemma 3.2, in a geometrical sense. That is, while the hypotheses of Lemma 3.2 allow a diversity of "shapes" in the graph of $u$ from $N-2$ to $N+1$, the following lemma requires that the graph be convex upward on the interval from $N$ to $N+3$.

LEMMA 3.3. If $u$ is a nontrivial solution of (1.1) and if

$$
\begin{aligned}
& \text { (a) } u_{n} \geqslant 0 \\
& \text { (b) } \Delta u_{n} \geqslant 0, \\
& \text { (c) } \Delta^{2} u_{n} \geqslant 0, \\
& \text { (d) } \Delta^{3} u_{n} \geqslant 0
\end{aligned}
$$

for some $n=N, N \geqslant 0$, then (3.6) holds for all $n \geqslant N$, with strict inequality in (3.6a, b, d) for all $n \geqslant N+3$, and in (3.6c) for all $n \geqslant N+4$. Furthermore

$$
\Delta^{4} u_{n} \geqslant 0 \text { for all } n \geqslant N \text {, }
$$

with strict inequality for $n \geqslant N+2$, and $u_{n}, \Delta u_{n}$, and $\Delta^{2} u_{n}$ all tend to $+\infty$ as $n \rightarrow \infty$.

Proof. Given a nontrivial solution $u$ of (1.1) satisfying (3.6) for $n=N \geqslant 0$, let $M=N+2$. Then $\Delta^{3} u_{M-2}=\Delta^{2} u_{M-1}-\Delta^{2} u_{M-2} \geqslant 0$, so

$$
\Delta^{2} u_{M-1} \geqslant \Delta^{2} u_{M-2}=\Delta^{2} u_{N} \geqslant 0 \text {. }
$$

Similarly, $\Delta^{2} u_{M-2} \geqslant 0$ implies $\Delta u_{M-1} \geqslant \Delta u_{M-2} \geqslant 0$, and $\Delta^{2} u_{M-1} \geqslant 0$ implies $\Delta u_{M} \geqslant \Delta u_{M-1}$, hence

$$
\Delta u_{M} \geqslant \Delta u_{M-1} \geqslant \Delta u_{M-2} \geqslant 0
$$

This in turn implies

$$
u_{M+1} \geqslant u_{M} \geqslant u_{M-1} \geqslant u_{M-2} \geqslant 0 .
$$

Thus $\Delta^{3} u_{M-2}, \Delta^{2} u_{M-1}, \Delta u_{M}$, and $u_{M}$ are all nonnegative, and it follows from Lemma 3.2 that
(a) $u_{n} \geqslant 0$,
(b) $\Delta u_{n} \geqslant 0$
(c) $\Delta^{2} u_{n-1} \geqslant 0$,
(d) $\Delta^{3} u_{n-2} \geqslant 0$,
(e) $\Delta^{4} u_{n-2} \geqslant 0$, 
for all $n \geqslant M$, with strict inequality in $(3.9 \mathrm{a}, \mathrm{e})$ for $n \geqslant M+2$, in (3.9b) for $n \geqslant M+1$, and in (3.9c, d) for $n \geqslant M+3$. Also $u_{n}, \Delta u_{n}$, and $\Delta^{2} u_{n}$ tend to $+\infty$ as $n \rightarrow \infty$. By shifting subscripts, and using $M=N+2$, we may rewrite (3.9) and the related statements about strict inequality as follows:
(a) $u_{n} \geqslant 0, n \geqslant N+2 \quad(>0$ for $n \geqslant N+4)$,
(b) $\Delta u_{n} \geqslant 0, n \geqslant N+2 \quad(>0$ for $n \geqslant N+3)$,
(c) $\Delta^{2} u_{n} \geqslant 0, n \geqslant N+1 \quad(>0$ for $n \geqslant N+4)$,
(d) $\Delta^{3} u_{n} \geqslant 0, n \geqslant N \quad$ (>0 for $n \geqslant N+3$ ),
(e) $\Delta^{4} u_{n} \geqslant 0, n \geqslant N \quad$ (>0 for $\left.n \geqslant N+2\right)$.

Since $M=N+2,(3.7)$ and (3.8) immediately imply that $u_{n} \geqslant 0$ and $\Delta u_{n} \geqslant 0$ hold also for $n=N+1$ and $n=N$. Furthermore, (3.8) implies that if $u_{N+3}=0$, then $u_{N+3}=u_{N+2}=u_{N+1}=u_{N}=0$, so $u$ would be the trivial solution of (1.1). Therefore $u_{N+3}>0$. Finally, $\Delta^{2} u_{N} \geqslant 0$ by hypothesis, and we have shown that all of conditions (3.6) hold for $n \geqslant N$, with strict inequality as stated in the conclusion.

We will need the following "backwards" version of Lemma 3.2, which describes behavior of a solution of (1.1) for values of $n$ less than some given $N$. Note that, in contrast to Lemmas 3.2 and 3.3, the sense of the inequalities alternates in conditions (3.11).

LEMMA 3.4. If $u$ is a nontrivial solution of (1.1) with
(a) $u_{n} \geqslant 0$
(b) $\Delta u_{n-1} \leqslant 0$,
(c) $\Delta^{2} u_{n-1} \geqslant 0$,
(d) $\Delta^{3} u_{n-1} \leqslant 0$,

for some $n=N \geqslant 3$, then $u$ satisfies (3.11) for all $n, 2 \leqslant n \leqslant N$, and

$$
\Delta^{4} u_{n-2} \geqslant 0, \quad 2 \leqslant n \leqslant N .
$$

Furthermore, $u_{0}>u_{1}>0$, and $\Delta u_{0}<0$. Strict inequality holds in (3.11a, e) for $2 \leqslant n \leqslant N-2$ (if $N \geqslant 4$ ), in (3.11b) for $2 \leqslant n \leqslant N-1$, and in (3.11c,d) for $2 \leqslant n \leqslant N-3$ (if $N \geqslant 5$ ).

Proof. Let $u$ be a nontrivial solution of (1.1) satisfying (3.11) for some $N \geqslant 3$. Let

$$
v_{j}=u_{2 N-j}, \quad j=0,1, \ldots, 2 N
$$


Then it is readily verified that

$$
\begin{array}{ll}
\Delta v_{j}=-\Delta u_{2 N-j-1}, & j=0,1, \ldots, 2 N-1, \\
\Delta^{2} v_{j}=\Delta^{2} u_{2 N-j-2}, & j=0,1, \ldots, 2 N-2, \\
\Delta^{3} v_{j}=-\Delta^{3} u_{2 N-j-3}, & j=0,1, \ldots, 2 N-3, \\
\Delta^{4} v_{j}=\Delta^{4} u_{2 N-j-4}, & j=0,1, \ldots, 2 N-4 .
\end{array}
$$

In particular, since $u$ is a solution of (1.1),

$$
\Delta^{4} v_{j-2}=\Delta^{4} u_{2 N-j-2}=b_{2 N-j} u_{2 N-j}, \quad j=2,3, \ldots, 2 N-2 .
$$

So $v$ is a solution of

$$
\Delta^{4} v_{j-2}=B_{j} v_{j}, \quad j=2,3, \ldots, 2 N-2,
$$

where $B_{j}=b_{2 N-j}>0, j=2,3, \ldots, 2 N-2$. Using (3.12) and the definition of $v_{j}$, we find that $v_{N}=u_{N}, \Delta v_{N}=-\Delta u_{N-1}=\Delta^{2} u_{N-1}, \Delta^{2} v_{N-1}$, and $\Delta^{3} v_{N-2}=-\Delta^{3} u_{N-1}$. It then follows from our hypotheses that $v$ satisfies the hypotheses of Lemma 3.2, which implies that
(a) $v_{j} \geqslant 0$,
(b) $\Delta v_{j} \geqslant 0$,
(c) $\Delta^{2} v_{j-1} \geqslant 0$,
(d) $\Delta^{3} v_{j-2} \geqslant 0$,
(e) $\Delta^{4} v_{j-2} \geqslant 0$,

for $N \leqslant j \leqslant 2 N-2$, with strict inequality holding as follows:
(a) $v_{j}>0$,
$N+2 \leqslant j \leqslant 2 N-2, \quad(N \geqslant 4)$,
(b) $\Delta v_{j}>0$
$N+1 \leqslant j \leqslant 2 N-2, \quad(N \geqslant 3)$,
(c) $\Delta^{2} v_{j-1}>0, \quad N+3 \leqslant j \leqslant 2 N-2, \quad(N \geqslant 5)$,
(d) $\Delta^{3} v_{j-2}>0, \quad N+3 \leqslant j \leqslant 2 N-2, \quad(N \geqslant 5)$,
(e) $\Delta^{4} v_{j-2}>0, \quad N+2 \leqslant j \leqslant 2 N-2, \quad(N \geqslant 4)$.

It follows from (3.12) and (3.14) that
(a) $u_{2 N-J} \geqslant 0$,
(b) $\Delta u_{2 N-j-1} \leqslant 0$,
(c) $\Delta^{2} u_{2 N-j-1} \geqslant 0$,
(d) $\Delta^{3} u_{2 N-j-1} \leqslant 0$,
(e) $\Delta_{2 N-J-2}^{4} \geqslant 0$, 
for $N \leqslant j \leqslant 2 N-2$, with strict inequality for the same values of $j$ as given in (3.15). If we let in $n=2 N-j$, we may rewrite (3.16) as
(a) $u_{n} \geqslant 0$,
(b) $\Delta u_{n-1} \leqslant 0$,
(c) $\Delta^{2} u_{n-1} \geqslant 0$,
(d) $\Delta^{3} u_{n-1} \leqslant 0$,
(e) $\Delta^{4} u_{n-2} \geqslant 0$,

for $2 \leqslant n \leqslant N$, which proves that (3.11) holds as claimed. The values of $n$ which give strict inequality in (3.17), as claimed in the conclusion of the lemma, follow immediately by letting $j=2 N-n$ in the $j$-intervals stated in (3.15). To complete the proof we need to extend the domain of $(3.17 \mathrm{a}, \mathrm{b})$ by showing $u_{0}>u_{1}>0$, and $\Delta u_{0}<0$. To do this, we first observe that (3.15b) implies $v_{2 N-1}-v_{2 N-2}=$ $\Delta v_{2 N-2}>0$ so

$$
u_{1}=v_{2 N-1}>v_{2 N-2} \geqslant 0 .
$$

Also, $v$ is a solution of (3.13) and we may rewrite (3.13), as in (2.2), as

$$
v_{j+2}=\Delta^{3} v_{j-2}+\Delta^{2} v_{j-1}+\Delta v_{j}+v_{j+1}+B_{j} v_{j} \text {. }
$$

In particular,

$$
v_{2 N}=\Delta^{3} v_{2 N-4}+\Delta^{2} v_{2 N-3}+\Delta v_{2 N-2}+v_{2 N-1}+B_{2 N-2} v_{2 N-2},
$$

from which it follows by $(3.14 \mathrm{~b}, \mathrm{c}, \mathrm{d})$ with $j=2 N-2$, and by (3.18), that

$$
u_{0}=v_{2 N}>0 \text {. }
$$

Similarly (3.14) and (3.20) imply

$$
\begin{aligned}
\Delta v_{2 N-1} & =v_{2 N}-v_{2 N-1} \\
& =\Delta^{3} v_{2 N-4}+\Delta^{2} v_{2 N-3}+\Delta v_{2 N-2}+B_{2 N-2} v_{2 N-2}>0,
\end{aligned}
$$

hence $\Delta u_{0}=-\Delta v_{2 N-1}<0$, hence $u_{1}<u_{0}$, which completes the proof.

We end this section with another lemma which describes the monotonicity of a solution which actually assumes the value zero. This result will be utilized extensively in the next section.

LeMMA 3.5. Let $N \geqslant 2$. If $u$ is a solution of (1.1) with $u_{N}=0, u_{N-1} \geqslant 0$, $u_{N+1} \geqslant 0, u_{N-1}$ and $u_{N+1}$ not both 0 , then at least one of the following conditions must be true.

(a) Either $u_{n}>0$ for all $n>N+1$, or

(b) $u_{n}>0$ for all $n<N-1, n \geqslant 0$.

In particular, $u$ cannot have generalized zeros of any order at both $R$ and $S$, where $R<N-1<N+1<S$. An analogous statement holds for the hypotheses $u_{N-1}$ $\leqslant 0$ and $u_{N+1} \leqslant 0$. 
Proof. From the hypotheses, we have

$$
\begin{aligned}
& u_{N}=0, \\
& \Delta u_{N}=u_{N+1}-u_{N}=u_{N+1} \geqslant 0, \\
& \Delta u_{N-1}=u_{N}-u_{N-1}=-u_{N-1} \leqslant 0, \\
& \Delta^{2} u_{N-1}=u_{N+1}-2 u_{N}+u_{N-1}>0 .
\end{aligned}
$$

If $\Delta^{3} u_{N-2} \geqslant 0$, then Lemma 3.2 applies, and we can conclude $u_{n}>0$ for all $n \geqslant N+2$. On the other hand, if $\Delta^{3} u_{N-2}<0$, then (1.1) implies $\Delta^{3} u_{N-1}-$ $\Delta^{3} u_{N-2}=\Delta^{4} u_{N-2}=b_{N} u_{N}=0$, hence $\Delta^{3} u_{N-1}=\Delta^{3} u_{N-2}>0$. If $N \geqslant 3$, then the hypotheses of Lemma 3.4 are satisfied, hence $u_{n}>0$ for all $n \leqslant N-2$.

If $N=2$, then $\Delta^{3} u_{N-2}=u_{3}-3 u_{2}+3 u_{1}-u_{0}$. Since $u_{N}=u_{2}=0$ and $\Delta^{3} u_{N-2}$ $=\Delta^{3} u_{N-1}<0$, we have that $u_{3}+3 u_{1}<u_{0}$. Since $u_{3}$ and $u_{1}$ are nonnegative and are not both equal to zero, it must be true that $u_{0}>0$, which is part (b) of our conclusion for $N=2$.

\section{Separation theorems}

In this section, we present several theorems dealing with the location and separation of zeros and generalized zeros. Before proceeding, we note again that the term "generalized zeros" includes both zeros and nodes, although we occasionally mention all three terms for emphasis.

THEOREM 4.1. If $u$ is a nontrivial solution of (1.1) with zeros at three consecutive values of $n$, say $N, N+1$ and $N+2$, then $u$ has no other generalized zeros. If $u_{N+3}>0(<0)$, then $\Delta u_{n} \geqslant 0(\leqslant 0)$ for all $n$, and the inequality is strict if $n \geqslant N+2$ or $n \leqslant N-1$. In particular, $p<N$ and $q>N+2$ imply $u_{p} u_{q}<0$.

Proof. Clearly $\Delta u_{N}=\Delta^{2} u_{N}=0$. Since $u$ is nontrivial, W.L.O.G. we may assume $u_{N+3}>0$. Thus $\Delta^{3} u_{N}=u_{N+3}>0$, and Lemma 3.3 implies $u$ is positive and strictly increasing for $n \geqslant N+3$.

Next, let $v=-u$. Then $v_{N+1}=0, \Delta v_{N}=0, \Delta^{2} v_{N}=0$ and $\Delta^{3} v_{N}<0$. If $N \geqslant 2$, then Lemma 3.4 implies that $v$ is positive and strictly decreasing for $0 \leqslant n \leqslant N$. (Note that $N$ in Lemma 3.4 is replaced here by $N+1$.) Thus $u$ is negative and strictly increasing for $0 \leqslant n \leqslant N$. If $N=1$, we again assume $u_{N+3}=u_{4}>0$. Then by (1.1), $\Delta^{4} u_{0}=b_{2} u_{2}=0$. But $\Delta^{4} u_{0}=u_{4}+u_{0}$ by (1.2), so $u_{0}=-u_{4}<0$ and $\Delta u_{0}=u_{1}-u_{0}>0$, as claimed. If $N=0$, the part of the conclusion concerning $n \leqslant N-1$ is vacuous, so this completes the proof. 
Notice that Theorem 4.1 confirms the remark made at the end of Section 2; i.e., the highest possible order of a generalized zero for a solution of (1.1) is 3 . Moreover, our next theorem implies that if a solution of (1.1) has a generalized zero of order 3, then it can have no other zeros or generalized zeros (except, of course, for the two adjacent zeros which are included in the definition of a generalized zero of order 3 ).

THEOREM 4.2. Let $N \geqslant 1$. Suppose $u$ is a solution of (1.1) with $u_{N}=0, u_{N+1}=0$, $u_{N+2} \neq 0$, but $N+2$ is a generalized zero for $u$. Then $u$ has no other generalized zeros. If $u_{N+2}>0(<0)$, then $\Delta u_{n} \geqslant 0(\leqslant 0)$ for all $n$, with strict inequality if $n \geqslant N+2$ or $n \leqslant N-1$. In particular, $p<N$ and $q>N+1$ imply $u_{p} u_{q}<0$.

Proof. Since $u_{N+2} \neq 0$, we may assume W.L.O.G. that $u_{N+2}>0$. Since $u_{N}=u_{N+1}=0, N+2$ cannot be a generalized zero of order 1 or 2 , and Theorem 4.1 implies that the order cannot be greater than 3. Thus $N+2$ is a generalized zero of order 3, which implies that $u_{N-1}<0$. From (1.1), we have

$$
\begin{aligned}
& u_{N+3}-4 u_{N+2}+6 u_{N+1}-4 u_{N}+u_{N-1}=b_{N+1} u_{N+1}, \text { or } \\
& u_{N+3}=4 u_{N+2}-u_{N-1} .
\end{aligned}
$$

This implies

$$
\begin{aligned}
\Delta^{3} u_{N} & =u_{N+3}-3 u_{N+2}+3 u_{N+1}-u_{N} \\
& =4 u_{N+2}-u_{N-1}-3 u_{N+2}+3 u_{N+1}-u_{N} \\
& =u_{N+2}-u_{N-1}>0 .
\end{aligned}
$$

Clearly, $\Delta^{2} u_{N}>0, \Delta u_{N}=0$ and $u_{N}=0$. We may now apply Lemma 3.3 and conclude that $u$ is positive and strictly increasing for $n \geqslant N+3$.

For $n \leqslant N$, let $v=-u$. Then $v_{N}=0, \Delta v_{N-1}<0, \Delta^{2} v_{N-1}>0$ and $\Delta^{3} v_{N-1}<0$. If $N \geqslant 3$, an application of Lemma 3.4 yields the result, just as in Theorem 4.1. If $N=2$, then $u_{2}=u_{3}=0, u_{1}<0, u_{4}>0$, and $\Delta u_{1}>0$. By (1.1) we have $\Delta^{4} u_{0}=$ $b_{2} u_{2}=0$. But

$$
\Delta^{4} u_{0}=u_{4}-4 u_{3}+6 u_{2}-4 u_{1}+u_{0}=u_{4}-4 u_{1}+u_{0},
$$

and so $4 u_{1}-u_{0}=u_{4}>0$. Hence $u_{0}<4 u_{1}<0$, and $u_{0}-u_{1}<3 u_{1}<0$. Therefore $u_{0}<0$ and $u_{0}>0$, as claimed. If $N=1$, then $u_{1}=u_{2}=0, u_{3} \neq 0$, and $N+2=3$ is a generalized zero. It follows from the definition of a generalized zero that this must be a generalized zero of order 3 , so that if $u_{3}>0$ then $u_{0}<0$. Hence $\Delta u_{0}>0$, which completes the proof.

COROLlaRY 4.1. If $u$ is a nontrivial solution of (1.1) with generalized zeros at $M$ and $P$ and a zero at $N, M+1<N<P-1$, then $u_{N-1} u_{N+1}<0$. In particular, $u$ does not have a generalized zero at $N+1$. 
Proof. Since $M+1<N<P-1$, it follows immediately from Theorem 4.1 that $u_{N+1}$ and $u_{N-1}$ cannot both equal 0 . If $u_{N+1} u_{N-1} \geqslant 0$, Lemma 3.5 implies that $u$ cannot have generalized zeros at both $M$ and $P$, a contradiction. Thus, $u_{N-1} u_{N+1}<0$.

COROLlaRY 4.2. If $u$ is a nontrivial solution of (1.1) with $u_{M}=u_{N}=u_{P}=0$, $M<N<P-1$, then $u_{N+1} \neq 0$.

Proof. If $N=M+1$, the corollary follows immediately from Theorem 4.1. If $N>M+1$, it follows from Corollary 4.1.

Corollary 4.3. If a nontrivial solution $u$ of (1.1) has $a$ zero at $M$ and $a$ generalized zero at $P$, where $M<P$, then $u$ cannot have consecutive zeros at $N$, $N+1$, where $M<N<P-1$.

Proof. Let $u$ be a nontrivial solution of (1.1) with zeros at $M, N, N+1$ and a generalized zero at $P$, where $M<N<P-1$. Theorems 4.1 and 4.2 imply that we must have $M<N-1$ and $P>N+2$, i.e., $M+1<N<P-1$. Corollary 4.1 implies $u_{N-1} u_{N+1}<0$, contradicting $u_{N+1}=0$.

We remark that Corollary 4.3 says that if a solution $u$ has four or more zeros, no two zeros can occur at consecutive values of $n$, unless they are the first two zeros or the last two zeros. For example, consider the sequence $\{-4,0,0,-1,0,1,0,0,4,15, \ldots\}$. This satisfies $\Delta^{4} u_{n-2}=5 u_{n}$, with $u_{1}=-4$. That this sequence is positive and increasing for $n \geqslant 9$ follows from Lemma 3.2 with $N=7$. Note also that the terms $u_{3}$ through $u_{7}$ illustrate Corollary 4.1.

THEOREM 4.3. If two nontrivial solutions $u$ and $v$ of (1.1) have three zeros in common, then $u$ and $v$ are linearly dependent, i.e., specifying any three zeros (not generalized zeros) uniquely determines a nontrivial solution up to a multiplicative constant.

Proof. We will consider two cases. First, assume $u_{M}=u_{N}=u_{N+1}=v_{M}=v_{N}$ $=v_{N+1}=0$, for some $M$ and $N, 0 \leqslant M<N$. Then $u_{N+2} \neq 0$ and $v_{N+2} \neq 0$ by Theorem 4.1. Define $w_{n}=v_{N+2} u_{n}-u_{N+2} v_{n}$. Since $w_{n}$ is a linear combination of $u_{n}$ and $v_{n}$, it is a solution of (1.1). However, $w_{M}=w_{N}=w_{N+1}=w_{N+2}=0$, and so $w$ must be the trivial solution by Theorem 4.1. Since $u_{N+2}$ and $v_{N+2}$ are nonzero, this means $u$ and $v$ are constant multiples of each other.

Next, suppose $u_{M}=u_{N}=u_{P}=0$ and $v_{M}=v_{N}=v_{P}=0$, where $M<N<P$ - 1. Then $u_{N+1} \neq 0$ and $v_{N+1} \neq 0$ by Corollary 4.3. Define $w_{n}=v_{N+1} u_{n}-$ $u_{N+1} v_{n}$. Clearly, $w_{M}=w_{N}=w_{N+1}=w_{P}=0$, which contradicts Corollary 4.2 
unless $w_{n} \equiv 0$. This means $u$ and $v$ are constant multiples of each other and completes the proof.

Our next theorem is a separation of zeros theorem for independent solutions of (1.1). When we say that $P$ and $Q, P<Q$, are consecutive generalized zeros of $u$, we mean that $u$ has no generalized zero $N$ such that $P<N<Q$.

THEOREM 4.4. Let $u$ and $v$ be nontrivial solutions of (1.1) with $u_{M}=v_{M}=u_{N}=$ $v_{N}=0$. Suppose $u$ has consecutive generalized zeros at $P$ and $Q$, where $M<P<Q$ $<N$. If $M+1<P$, then either

$$
\begin{gathered}
v_{P-1} v_{P}<0, \quad \text { or } \\
v_{k} v_{k+1} \leqslant 0 \text { for some } k, P \leqslant k \leqslant Q-1 .
\end{gathered}
$$

If $M+1=P$ and $u_{P}=0$, then (4.2) holds. If $M+1=P$ and $u_{P} \neq 0$, but $P$ is $a$ generalized zero for $u$, then either (4.2) holds, or

$$
v_{P} \neq 0 \text { and } v \text { has a generalized zero at } P \text {. }
$$

In any case, therefore, $v$ has a generalized zero for some $Z$, where $P \leqslant Z \leqslant Q$.

Proof. Consider the generalized zero of $u$ at $P$. Then (2.4), (2.5) or (2.6) must hold for $n=P$.

First, assume that (2.4) holds, i.e., $u_{P}=0$, and suppose $P-1>M$. Then we must have $u_{P-1} u_{P+1} \neq 0$, otherwise we contradict Corollary 4.3. If $u_{P-1} u_{P+1}>0$, then Lemma 3.5 is contradicted. Thus $P-1>M$ implies $u_{P-1} u_{P+1}<0$. This is listed as possibility (4.5) below. If $P-1=M$, then $u_{P-1}=u_{P}=0$, and $P+1$ is not a generalized zero, since otherwise either Theorem 4.1 or Theorem 4.2 would contradict the fact that $u_{N}=0$. This is listed as (4.6) below.

Next, if (2.5) holds, then $u_{P-1} u_{P}<0$, hence $P-1>M$ and condition (4.4) below must hold.

Finally, suppose that (2.6) holds. If $k=3$ (the highest value allowed, by the remark following Theorem 4.1), there would be two consecutive zeros of $u$ followed by a generalized zero. Theorem 4.2 implies $u_{N} \neq 0$, a contradiction. Thus $P$ cannot be a generalized zero of order 3. Now suppose $k=2$. If $P-2>M$, we may assume $u_{P}>0, u_{P-1}=0$ and $u_{P-2}>0$. Lemma 3.5 then implies that $u_{M}$ and $u_{N}$ cannot both equal 0 , a contradiction. If $P-2=M$ and $k=2$, then $u_{M} \neq 0$, a contradiction. Suppose $P-2<M$. Then $P=M+1$, $u_{P-1}=0$ and $u_{P} u_{P-2}=u_{P} u_{M-1}>0$. This possibility can occur and is listed as (4.7) below.

Therefore, if $u$ has a generalized zero at $P$, one of the following occurs:

$$
\begin{gathered}
M+1<P \text { and } u_{P-1} u_{P}<0, \\
M+1<P, \quad u_{P}=0, \text { and } u_{P-1} u_{P+1}<0, \\
M+1=P, \quad u_{P}=0, \text { and } P+1 \text { is not a generalized zero, or }
\end{gathered}
$$




$$
M+1=P, \quad u_{P} \neq 0 \text { and } u_{P} u_{M-1}>0 .
$$

A similar analysis holds for the generalized zero at $Q$. However, it will be sufficient for us to note that if $u_{Q-1} u_{Q}>0$ then $Q$ cannot be a generalized zero, so it must be true that

$$
u_{Q-1} u_{Q} \leqslant 0 .
$$

Suppose that (4.4) holds for $u$, and neither (4.1) nor (4.2) is true for $v$. W.L.O.G., we may assume $u_{P-1}>0$ and $u_{P}<0$. Then $u_{n}<0$ for $P \leqslant n \leqslant Q-1$, since $P$ and $Q$ are consecutive generalized zeros for $u$. We may also assume $v_{P-1} \geqslant 0$ and $v_{n}>0$ for $P \leqslant n \leqslant Q$. Let $w_{n}$ be defined by:

$$
w_{n}=v_{n} u_{n-1}-u_{n} v_{n-1} \text {. }
$$

Clearly, $w_{P}>0$. Consider $w_{Q}$. If $w_{Q}>0$, since $v_{Q}>0, v_{Q-1}>0$, and $u_{Q-1}<0$, we must conclude $u_{Q}<0$. However, if $u_{Q}$ and $u_{Q-1}$ are both negative, $u$ does not have a generalized zero at $Q$. Thus, $w_{Q} \leqslant 0$. Since $w_{P}>0$ and $w_{Q} \leqslant 0$, there exists a first integer $R, P \leqslant R \leqslant Q-1$, such that $w_{R}>0$ and $w_{R+1} \leqslant 0$. Let $s_{n}$ be a solution of (1.1) defined by

$$
s_{n}=v_{R} u_{n-1}-u_{R} v_{n-1} .
$$

Note that $s_{R}=w_{R}>0, s_{R+1}=0$ and $s_{R+2}=-w_{R+1} \geqslant 0$. Then Lemma 3.5 implies that $s_{M+1}$ and $s_{N+1}$ cannot both equal 0 , a contradiction. Thus, if (4.4) holds for $u$, then $v$ satisfies (4.1) or (4.2), so $v$ has a generalized zero for some $Z$, $P \leqslant Z \leqslant Q$.

Suppose now that (4.5) holds for $u$ and that neither (4.1) nor (4.2) is true for $v$. W.L.O.G., assume $u_{P-1}>0$ and $u_{P+1}<0$. We may also assume $u_{n}<0$ for $P+1 \leqslant n \leqslant Q-1$ and $v_{n}>0$ for $P \leqslant n \leqslant Q$. Define $w$ as in (4.9). Then $w_{P}>0$. Arguing as we did above, we again arrive at a contradiction.

Suppose that (4.6) holds for $u$ and that (4.2) is not true for $v$. Then we may assume $v_{n}>0, P \leqslant n \leqslant Q$, where $P-1=M$. W.L.O.G., assume $u_{P+1}<0$. Then $w_{P+1}>0$. An application of the same argument as above, with $P$ replaced by $P+1$, leads to a contradiction.

Finally, suppose that (4.7) holds for $u$ but that neither (4.2) nor (4.3) is true for $v$. Then we may assume $u_{n}<0$ for $P \leqslant n \leqslant Q-1, u_{M+1}<0$, and $v_{n}>0$ for $P \leqslant n \leqslant Q$, and $M+1$ is not a generalized zero for $v$. Since $v_{M}=0$, it follows from the definition of a generalized zero that $v_{M-1} \leqslant 0$. As before, define $w_{n}$ by (4.9). Clearly $w_{P}=0$, so we consider $w_{P+1}$. Suppose $w_{P+1}>0$. As above, we can then conclude that $w_{Q} \leqslant 0$, and the same argument as in the preceding case leads to a contradiction. Next, assume $w_{P+1} \leqslant 0$. Let $s_{n}$ be a solution of (1.1) defined by

$$
s_{n}=v_{P} u_{n-1}-u_{P} v_{n-1} .
$$

Then $s_{M+1}=0$ and $s_{M+2}=0$ since $P=M+1$, and $s_{M+3}=-w_{P+1} \geqslant 0$. If $w_{P+1}=0$, the solution $s$ has three consecutive zeros at $M+1, M+2, M+3$ and 
another zero at $N+1$, a contradiction of Theorem 4.1. Thus $w_{P+1}<0$ and therefore $s_{M+3}>0$. This means that $s_{M}>0$, because if $s_{M} \leqslant 0$ Theorem 4.1 or Theorem 4.2 would again contradict $s_{N+1}=0$. However, $s_{M}>0$ implies $v_{P} u_{M-1}$ $>u_{p} v_{M-1}$. Since $v_{M-1} \leqslant 0$ and $u_{p}<0$, this means $v_{p} u_{M-1}>0$. This is a contradiction, since $v_{P}>0$ and $u_{M-1}<0$. This proves the theorem.

Concerning the hypotheses of Theorem 4.4, it seems reasonable to ask for two solutions $u_{n}$ and $v_{n}$ both to have a zero at some specified value $M$; i.e. $u_{M}=v_{M}=$ 0 . However, examples indicate it may not always happen in this case that there exists an $N>M$ such that $u_{N}=v_{N}=0$. It might be useful, therefore, to allow at least one of the solutions, say $v$, to have a generalized zero at $N>M$. Given $M$ and $N>M+2$, let $u$ be a solution such that $u_{M}=u_{N-1}=u_{N}=0$. It is easy to argue that such a $u$ exists, as follows. Let $r$ be a solution of (1.1) such that $r_{N-z}=r_{N-1}=r_{N}=0$ and $r_{N+1}>0$. Let $s$ be a solution such that $s_{N-2}<0$, $s_{N-1}=s_{N}=0$ and $s_{N+1}>0$. Theorems 4.1 and 4.2 apply to $r$ and $s$, respectively, and a suitable linear combination of them yields $u$. By Theorem $4.3, u$ is essentially unique. We can now state the following separation theorem.

THEOREM 4.5. Suppose $v$ is a nontrivial solution of (1.1) such that $v_{M}=0$ and $v$ has a generalized zero at $N>M+2$. Let $u$ be the (essentially unique) solution such that $u_{M}=u_{N-1}=u_{N}=0$. Suppose $u$ has consecutive generalized zeros at $P$ and $Q$, where $M<P<Q<N$. Then the same conclusions hold as in Theorem 4.4, so that $v$ has a generalized zerc at $Z$, where $P \leqslant Z \leqslant Q$. Similarly, if $v$ has consecutive generalized zeros at $P$ and $Q$, then $u$ has a generalized zero for some $Z, P \leqslant Z \leqslant Q$.

Proof. Aside from one or two differences, the proof is essentially the same as that for Theorem 4.4. We arrive at (4.4), (4.5), (4.6), or (4.7) for the generalized zero of $u$ at $P$. At $Q, u$ satisfies (4.8).

Suppose that (4.4) holds for $u$ and neither (4.1) nor (4.2) is true for $v$. We can again assume $u_{n}<0$ for $P \leqslant n \leqslant Q-1$. Define $w_{n}$ as in (4.9). Arguing as before, we find there exists an $R, P \leqslant R \leqslant Q-1$, such that $w_{R}>0$ and $w_{R+1} \leqslant 0$. Let $s_{n}$ be defined by (4.10). Then $s_{R}=w_{R}>0, s_{R+1}=0$, and $s_{R+2}=-w_{R+1} \geqslant 0$. We must next consider the following two possibilities (which is not necessary in Theorem 4.4, since there $v_{N}=0$, but here $N$ is a generalized zero for $v$ ).

Possibility 1: Let $R+1=N-1$, in which case $R+1=Q=N-1$, and $s_{R+2}=-w_{R+1}=-w_{N-1}=-v_{N-1} u_{N-2} \geqslant 0$. If $-w_{R+1}=0$, since $u_{N-2}<0$ we have $v_{N-1}=0$, a contradiction. If $-w_{R+1}>0$, since $Q=N-1$ and $u_{N-2}<0$, we must have $v_{N-1}>0$. Also, $s_{R+3}=v_{R} u_{R+2}-u_{R} v_{R+2}=v_{R} u_{N}-u_{R} v_{N}=$ $-u_{R} v_{N}$. Since $s_{M+1}=0$, Lemma 3.5 implies $s_{R+3}=-u_{R} v_{N}>0$. Since $u_{R}<0$, we have $v_{N}>0$. Thus $v_{N}$ and $v_{N-1}$ are both positive, which contradicts the fact that $v$ has a generalized zero at $N$. 
Possibility 2: Let $R+1<N-1$. We again have $s_{R}>0, s_{R+1}=0$ and $s_{R+2}=$ $-w_{R+1} \geqslant 0$. Since $s_{M+1}=0$, Lemma 3.5 implies that $s_{n}>0$, for all $n>R+2$. In particular, $s_{N}>0$ and $s_{N+1}>0$. However, $s_{N}=v_{R} u_{N-1}-u_{R} v_{N-1}=-u_{R} v_{N-1}$, and $s_{N+1}=v_{R} u_{N}-u_{R} v_{N}=-u_{R} v_{N}$. Since $s_{N}>0, s_{N+1}>0$ and $u_{R}<0$, we conclude that $v_{N-1}$ and $v_{N}$ are both positive, again contradicting the assumption that $v$ has a generalized zero at $N$. Thus we have shown that if (4.4) holds for $u$, then $v$ satisfies (4.1) or (4.2).

Next, suppose that (4.5) holds for $u$ and that neither (4.1) nor (4.2) is true for $v$. We may assume $u_{P-1}>0$ and $u_{P+1}<0$. Just as in Theorem 4.4, we can argue that $w_{P}>0$. This means there is a first integer $R$ such that $w_{R}>0$ and $w_{R+1}<0$. Arguing as we did in the previous three paragraphs, we arrive at a contradiction.

Suppose (4.6) holds for $u$ and (4.2) is not true for $v$. As in Theorem 4.4, we can argue that $w_{P+1}>0$. Applying the same argument as we did for (4.4) and (4.5) in the preceding parts of this proof yields a similar contradiction.

Finally, suppose (4.7) is true for $u$ but that neither (4.2) nor (4.3) is true for $v$. Then we may assume $u_{n}<0, P \leqslant n \leqslant Q-1, u_{M-1}<0, v_{n}>0, P \leqslant n \leqslant Q$, and $v_{M-1} \leqslant 0$. Define $w_{n}$ by (4.9). Clearly $w_{P}=0$. If $w_{P+1}>0$, we reach the same contradiction as we did for (4.4), (4.5) and (4.6). Thus, we assume $w_{P+1} \leqslant 0$. Let $s$ be the solution defined by (4.11). Then $s_{M+1}=0, s_{M+2}=0$ and $s_{M+3}=-w_{P+1}$ $\geqslant 0$. If $-w_{P+1}=0, s$ has three consecutive zeros at $M+1, M+2$ and $M+3$. Theorem 4.1 implies $s$ is one sign, say positive, for all $n \geqslant M+3$. In particular, $s_{N}=-u_{M+1} v_{N-1}>0$ and $s_{N+1}=-u_{M+1} v_{N}>0$. This implies $v_{N-1} v_{N}>0$, a contradiction. Therefore, we assume $-w_{P+1}>0$ so that $s_{M+3}>0$. If $s_{M} \leqslant 0$, Theorems 4.1 or 4.2 imply $s$ is of one sign for all $n \geqslant M+3$ and we can again arrive at the contradiction $v_{N-1} v_{N}>0$. Thus, $s_{M}>0$, which implies $v_{P} u_{M-1}>$ $u_{P} v_{M-1}$. Since $u_{P}<0$ and $v_{M-1} \leqslant 0$, we have $v_{P} u_{M-1}>0$. This is a contradiction since $v_{P}>0$ and $u_{M-1}<0$. Thus $v_{n}$ must have a generalized zero for some $Z$, $P \leqslant Z \leqslant Q$.

If we assume $v$ has consecutive generalized zeros at $P$ and $Q$ and try to show $u$ has a generalized zero for some $Z, P \leqslant Z \leqslant Q$, essentially the same proof works. We omit the details.

\section{Recessive solutions}

Certain types of second order linear difference equations have so-called recessive solutions [9]. Under certain conditions, it can be shown that these solutions are positive and monotone decreasing [10]. Recessive solutions for second order equations have been found useful for calculating certain types of special functions [9]. In this section, we define recessive solutions for the fourth order linear difference equation (1.1) and discuss some associated properties. 
Definition. $A$ solution $u$ of (1.1) will be called recessive if there exists an $M \geqslant 0$ such that

$$
u_{n}>0, \Delta u_{n} \leqslant 0, \quad \Delta^{2} u_{n} \geqslant 0 \text { and } \Delta^{3} u_{n} \leqslant 0, \quad n \geqslant M .
$$

We will construct a recessive solution of (1.1) by modifying the technique used in the second order case ([6], [11]). Let $u^{k}=\left\{u_{n}^{k}\right\}$ be the solution of (1.1) such that $u_{k}^{k}=u_{k+1}^{k}=u_{k+2}^{k}=0$ and $u_{0}^{k}=1, k \geqslant 1$. For each $k, u^{k}$ exists and is unique. The existence is clear from Theorem 4.1 and a normalization, while the uniqueness follows from Theorem 4.3. Note that by construction,

$$
0 \leqslant u_{n}^{k} \leqslant 1, \quad 0 \leqslant n \leqslant k+2 .
$$

Also, Theorem 4.1 implies that

$$
u_{j}^{k} \geqslant u_{j+1}^{k}, \quad j \geqslant 0 .
$$

We now consider $u_{1}^{k}$ as a sequence in $k$. By (5.2), $0 \leqslant u_{1}^{k} \leqslant 1$, for all $k$. Thus $\lim \sup _{k \rightarrow \infty}\left\{u_{1}^{k}\right\}$ exists; call it $u_{1}$. Then there exists a subsequence $k_{1 i}$ of $k$ such that $u_{1}^{k_{1}} \rightarrow u_{1}$ as $i \rightarrow \infty$. Next, consider $u_{2}^{k}$ as a sequence in $k$. By (5.2), $\lim \sup _{i \rightarrow \infty} u_{2}^{k_{1 i}}$ exists; call it $u_{2}$. Also, there exists a subsequence $k_{21}$ of $k_{1 i}$ such that $u_{21}^{k_{21}} \rightarrow u_{2}$ (and $u_{1}^{k_{21}} \rightarrow u_{1}$ ) as $i \rightarrow \infty$. In a similar fashion, by considering $u_{3}^{k}$, we can arrive at a subsequence $k_{3}$ and a limit $u_{3}$ such that $u_{j}^{k_{3 i}} \rightarrow u_{j}$ as $i \rightarrow \infty$, $1 \leqslant j \leqslant 3$. Clearly $u_{0}^{k_{3 i}}=1$, for all $i$.

Recall that by definition, for any $n$ and any $k$,

$$
u_{n+2}^{k}-4 u_{n+1}^{k}+6 u_{n}^{k}-4 u_{n-1}^{k}+u_{n-2}^{k}=b_{n} u_{n}^{k} .
$$

Consider (5.4) with $n=2$ and $k$ replaced by $k_{3 i}$. We can conclude $\lim _{i \rightarrow \infty} u_{4}^{k_{3 t}}$ exists; call it $u_{4}$. Now replace $n$ by 3 in (5.4) and conclude the existence $\lim _{i \rightarrow \infty} u_{5}^{k_{3 i}} ;$ call the limit $u_{5}$. Proceeding inductively, we conclude that $\lim _{i \rightarrow \infty} u_{n}^{k_{31}}$ exists for any $n \geqslant 0$, and we call the limit $u_{n}$. Replacing $k$ by $k_{3}$ in (5.4) and letting $i \rightarrow \infty$, we can conclude that $u_{n}$ is a solution of (1.1). Also,

$$
u_{n} \geqslant u_{n+1} \geqslant 0 \text {. }
$$

This follows from (5.3) by replacing $k$ by $k_{3}$, fixing $j$, and letting $i \rightarrow \infty$. From (5.5), we can conclude that

$$
\lim _{n \rightarrow \infty} u_{n} \text { exists; call it } L \text {. }
$$

We will now show $u$ is a recessive solution of (1.1).

LEMMA 5.1. The solution $u$ constructed above is a recessive solution of (1.1). In addition, $\Delta u_{n}, \Delta^{2} u_{n}$ and $\Delta^{3} u_{n}$ all monotonically approach zero as $n \rightarrow \infty$.

Proof. We first show(5.1) is satisfied. By (5.3) and Theorem 4.1, $u_{k_{31}+3}^{k_{3 t_{1}}}<0$. Choose $k_{3 i} \geqslant 3$ and apply Lemma 3.4 with $N=k_{3 i}+1$. We can conclude that 
for any $n$ such that $2 \leqslant n \leqslant k_{3 i}+1, \Delta u_{n-1}^{k_{33}} \leqslant 0, \Delta^{2} u_{n-1}^{k_{33}} \geqslant 0$ and $\Delta^{3} u_{n-1}^{k_{34}} \leqslant 0$. Letting $i \rightarrow \infty$ implies $u$ satisfies (5.1) for $M=1$ and is recessive. We remark that $u$ also satisfies (5.1) for $M=0$. Concerning the monotonicity, choose any $n \geqslant 2$ and any $k_{3 i} \geqslant n$. Then $\Delta^{2} u_{n-1}^{k_{3 i}} \geqslant 0$, which means $\Delta u_{n}^{k_{3 i}} \geqslant u_{n-1}^{k_{3 i}}$, hence $0 \leqslant-\Delta u_{n}^{k_{3 n}}$ $\leqslant-\Delta u_{n-1}^{k_{3 i}}$. Taking the limit as $i \rightarrow \infty$ implies that $\Delta u_{n}$ is monotonically decreasing in absolute value. By (5.6), since $u_{n}$ monotonically approaches a finite limit, $\Delta u_{n} \rightarrow 0$ as $n \rightarrow \infty$. The argument that $\Delta^{2} u_{n}$ and $\Delta^{3} u_{n}$ monotonically approach zero is similar and will be omitted.

Using Lemma 5.1 and (1.1), we may deduce the following formulas for this recessive solution $u_{n}$.

$$
\begin{aligned}
& -\Delta^{3} u_{n-2}=\sum_{j=n}^{\infty} b_{j} u_{j} . \\
& \Delta^{2} u_{n-2}=\sum_{j=n}^{\infty}(j-n+1) b_{j} u_{j} . \\
& -\Delta u_{n-2}=\sum_{j=n}^{\infty}[(j-n+1)(j-n+2) / 2] b_{j} u_{j} . \\
& u_{n-2}=L+\sum_{j=n}^{\infty}[(j-n+1)(j-n+2)(j-n+3) / 6] b_{j} u_{j} .
\end{aligned}
$$

LEMMA 5.2. If $\sum^{\infty} n^{3} b_{n}=\infty$, then the recessive solution $u$ constructed above approaches zero as $n \rightarrow \infty$.

Proof. Since $u$ is positive and monotone decreasing, the result follows directly from (5.10).

In the second order case, it is known that this recessive solution $u$ is unique, once the starting value $u_{0}$ is specified [9]. It would be interesting to know under what circumstances uniqueness holds for recessive solutions of (1.1). A lemma that may be of some use in this respect is the following.

LEMMA 5.3. Suppose $w$ and $u$ are two recessive solutions of (1.1) such that $w_{M}=u_{M}$. If $w_{n} \geqslant u_{n}$ for all $n \geqslant M$, then $w_{n} \equiv u_{n}$.

Proof. Let $W=\lim _{n \rightarrow \infty} w_{n}$ and $L=\lim _{n \rightarrow \infty} u_{n}$. By hypothesis, $W \geqslant L$. Next, define $y_{n}=w_{n}-u_{n}$. From (5.10) with $n=M+2$ we have

$$
0 \geqslant W-L+\sum_{j=M+2}^{\infty}[(j-1) j(j+1) / 6] b_{j} y_{j} \geqslant 0 .
$$

From this we can conclude $w_{n} \equiv u_{n}$. 


\section{References}

[1] G. Dahlquist, "Stability and error bounds in the numerical integration of ordinary differential equations", Kungl. Tekn. Högsk. Handl. Stockholm 130 (1959).

[2] T. Fort, Finite differences and difference equations in the real domain (Oxford University Press, London, 1948).

[3] H. I. Freedman, Deterministic mathematical models in population ecology (Marcel Dekker, Ncw York, 1980).

[4] D. Greenspan, Discrete models (Addison-Wesley, Reading, Massachusetts, 1973).

[5] P. Hartman, "Difference equations: disconjugacy, principal solutions, Green's functions, complete monotonicity", Trans. Amer. Math. Soc. 246 (1978), 1-30.

[6] P. Hartman and A. Wintner, "On linear difference equations of the second order", Amer. J. Math. 72 (1950), 124-128.

[7] P Henrici, Discrete variable methods in ordinary differential equations (Wiley, New York, 1962).

[8] W. Leighton and Z. Nehari, "On the oscillation of solutions of self-adjoint linear differential equations of the fourth order", Trans. Amer. Math. Soc. 89 (1958), 325-377.

[9] F. W. J. Olver and D. J. Sookne, "Note on backward recurrence algorithms", Math. Comp. 26 (1972), 941-947.

[10] W. T. Patula, "Growth and oscillation properties of second order linear difference equations", SIA M J. Math. Anal. 10 (1979), 55-61.

[11] W. T. Patula, "Growth, oscillation and comparison theorems for second order linear difference equations", SIAM J. Math. Anal. 10 (1979), 1272-1279.

[12] J. H. Van Lint, Introduction to coding theory (Springer-Verlag, New York, 1982). 